Revue d'histoire de l'enfance « irrégulière »

Le Temps de l'histoire

Hors-série | 2001

Histoire et justice, panorama de la recherche

\title{
Les complaintes judiciaires
}

Joseph Le Floc'h

\section{OpenEdition}

Journals

Édition électronique

URL : http://journals.openedition.org/rhei/421

DOI : 10.4000/rhei.421

ISBN : 978-2-7535-1641-0

ISSN : 1777-540X

Éditeur

Presses universitaires de Rennes

Édition imprimée

Date de publication : 15 novembre 2001

Pagination : 93-103

ISSN : 1287-2431

Référence électronique

Joseph Le Floc'h, « Les complaintes judiciaires », Revue d'histoire de l'enfance « irrégulière » [En ligne], Hors-série | 2001, mis en ligne le 31 mai 2007, consulté le 19 avril 2019. URL : http://

journals.openedition.org/rhei/421; DOI : 10.4000/rhei.421

(c) PUR 


\section{Chanteurs de rue et complaintes judiciaires}

Joseph Le Floc'h ${ }^{(2)}$

\section{Quelques remarques}

\section{à propos des complaintes françaises}

Dès le XVIème siècle, et parallèlement au succès des occasionnels, ${ }^{(2)}$ on sait que la complainte répond à une attente et rencontre son public. Pierre de l'Estoile, par exemple, écrit qu'à la mort de Marie Stuart, en 1587 : « les pasquils [complaintes], placcards, tombeaux et discours sur cette mort violente voloient à Paris et partout $\gg .^{(3)}$

Tous les témoignages confirment en effet que sous l'Ancien Régime les chansons imprimées sur feuilles volantes sont monnaie courante. La figure du chanteur ambulant apparaît d'ailleurs systématiquement dans les évocations du paysage sonore parisien. Toujours mentionné par exemple dans les cris de Paris, ce digne héritier du jongleur médiéval fait régulièrement commerce de pièces de circonstance, rapportant tel ou tel événement politique ou militaire, célébrant la naissance ou l'anniversaire d'un membre de la famille royale ou, plus simplement encore, son combat victorieux contre la maladie. Mais il lui revient également d'assurer la diffusion de bien d'autres registres : cantiques, noëls, chansons gaillardes, chants à boire et complaintes. Tous ces genres doivent beaucoup à ce mode de propagation où oralité et écriture se conjuguent avec une grande efficacité et concernent tant les milieux citadins que ruraux.

Dans son célèbre Tableau de Paris ${ }^{(4)}$ parus en 1788, Louis-Sébastien Mercier, brosse ainsi quelques portraits de chanteurs ambulants et l'acuité de son témoignage est aujourd'hui fort précieuse :

"Un parricide, un empoisonneur, un assassin, le lendemain, que dis-je, dès le jour même de leur supplice, enfantent des complaintes qui sont chantées dans tous les carrefours et composées par les chanteurs du Pont-Neuf.(5) "

Par cette observation, l'auteur met en évidence deux éléments caractéristiques de ce genre.
(1) Professeur de musicologie, université de Poitiers

(2) Livrets imprimés à l'occasion de faits divers sensationnels, et donc sans périodicité, d'où l'appellation. Cette tradition est inaugurée en France dès la fin du XVème siècle.

(3) Cité par Jean-Pierre SEGUIN, Nouvelles à sensation. Canards $d u$ XIXème siècle, Paris, Colin, 1959.

(4) Louis-Sébastien MERCIER, Tableau de Paris, Amsterdam, 17811788.

(5) Tableau de Paris (extraits), Paris, Horizons de France, 1947, p. 156.

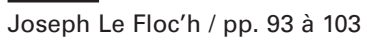


(6) Souscription pour une Histoire générale du Pont-Neuf, Londres, 1750, BN $8^{\circ} \mathrm{Z}$. Le Senne, 6556, p. 33.
- D’une part la complainte criminelle a pour fonction essentielle de clôturer une affaire judiciaire. Ce qu'elle rapporte en effet, c'est bien une entité indissociable qui, partant d'un fait négatif, le crime, s'impose un retour à une situation positive par la voie du supplice et de l'expiation.

- D'autre part ce sont les chanteurs du Pont-Neuf qui assurent la composition de telles pièces. Qu'est-ce à dire exactement ? Certes, depuis le XVIIème siècle, le Pont-Neuf constitue le centre névralgique de la vie musicale populaire parisienne. En 1750, un contemporain rapporte que "Le Pont-Neuf est le centre de gravité de notre musique. Il faut qu'après un règne ou plus long, ou plus court, et plus ou moins étendu, elle retombe sur le PontNeuf. ${ }^{(6)}$ " Chanter sur le pont ou sur les quais adjacents constitue alors un véritable métier ; les évocations sont nombreuses ; les représentations le sont également. Le chanteur se présente assez rarement seul ; plus souvent il apparaît en binôme : chanteur et vendeur, chanteur et instrumentiste. Tous deux sont fréquemment juchés sur des tréteaux. Ainsi visibles et audibles, ils délimitent un espace de sociabilité dans lequel pourra s'actualiser le message sonore. Quel que soit le lieu où il se produit, le chanteur ne manque jamais de concurrence puisque, bien évidemment, il sélectionne les lieux de forte affluence. Alors, attirer, retenir, donner à entendre et enfin, vendre, ainsi pourrait-on résumer les quelques étapes obligées de ce circuit commercial. Au terme de cette rencontre, l'objet échangé sera un papier de fort mauvaise qualité, le plus souvent un simple feuillet, parfois plusieurs, sommairement reliés sans couverture.

Chanter dans la rue n'est pas une activité clandestine loin s'en faut, hormis les temps de crise, celles de la Fronde ou de la Révolution par exemple. Plus souvent, et en se donnant à voir et à entendre, le chanteur montre au contraire qu'il est directement lié au(x) pouvoir(s). Soumis à censure, à permis d'imprimer, il ne peut guère refuser d'être porte-voix et porte-parole si on le lui demande.

Pourtant, le diseur de complaintes jouit, semble-t-il, d'un statut particulier dans cet espace de diffusion. Louis-Sébastien Mercier rapporte en effet que :

"Ces chanteurs se distinguent des chantres profanes, et pour marquer leur métier religieux, ils portent une croix et un scapulaire [...] Jamais ils ne montent 
sur un tréteau, ils marchent à pied d'un pas lent, et leur extérieur annonce la componction. ${ }^{(7)}$ "

Cette précision est intéressante en ce qu'elle révèle bien la nature d'une intention fondamentalement didactique. On ne donne pas à entendre la complainte comme une chanson légère, ou comme la célébration d'une victoire. La complainte criminelle s'entonne un peu à l'écart de la fête, au même titre que le crime s'écarte d'une conduite usuelle et acceptable. La complainte procède à bien des égards d'une véritable liturgie où la croix et le scapulaire sont en phase avec le genre littéraire le plus fréquemment sollicité : la confession.

C'est sur ce mode, et dans le cadre habituel d'un triptyque, que se présentent un grand nombre de complaintes judiciaires de l'Ancien Régime. Une formule d'appel suivie d'une narration principale, une brève morale de l'événement, ainsi peut-on résumer le scénario de base d'une telle composition. A bien des égards, il emprunte donc au schéma usuel d'une composition théâtrale en trois étapes : prologue, action, épilogue.

A elle seule, la formule d'appel donne parfois quelque indication sur le retentissement de l'événement, ou tout au moins sur le degré d'exemplarité que l'on veut lui associer. Si l'on convoque par exemple assez simplement "les braves bourgeois de Merville » dans la complainte sur l'exécution de Pierre Antoine Hugues, en 1705, c'est " au peuple de France, d'Artois, de Flandres et d'autres endroits » que l'on s'adresse en 1721, lors du supplice de Cartouche. Roué vif en place de Grève, au terme d'une carrière de bandit particulièrement célèbre sur le Pont-Neuf, il est vrai que sa réputation avait depuis longtemps franchi les portes de la capitale.

Une longue narration expose ensuite le (ou les) crime(s), avant l'énoncé puis l'exécution de la sentence. Devant la mort, il est d'usage de rapporter comment le supplicié s'est présenté devant Dieu : contrit ou muré dans le silence, bon ou mauvais larron, on attend de lui qu'il veuille bien tenir son rôle jusqu'à son terme. Dès lors, les conditions de sa mort peuvent donner lieu à un bref épilogue : édifiant, si le condamné s'est repenti, plus menaçant s'il n'a laissé paraître aucun remords. Mais toujours, cette conclusion aura valeur d'avertissement, principalement destiné à la jeunesse.

(7) Cité par Claude DUNETON, Histoire de la chanson française, Paris, Seuil, 1998, tome I, p. 889. 


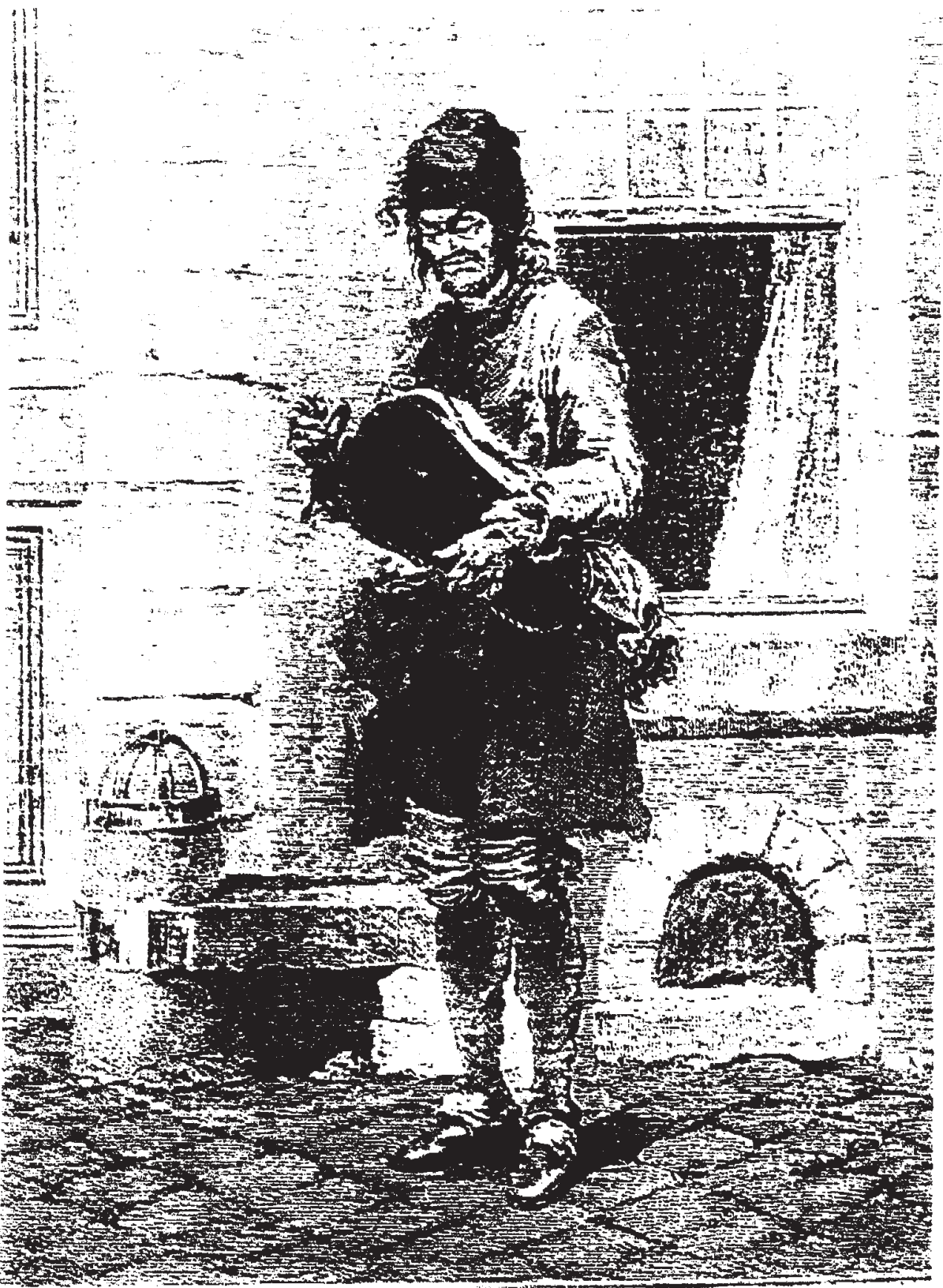

Illustration 1 
Michel Leclerc, le vielleur du Pont-Neuf, fut probablement au XVIIIème siècle le plus célèbre de ces auteurs spécialistes des complaintes des suppliciés. Il a chanté par exemple la condamnation de Damiens, écartelé en 1757 en place de Grève pour avoir porté un coup de canif à Louis XV. En 1777, il a chanté également le supplice de Desrues, accusé d'empoisonnement et exécuté en place de Grève le 6 mai. Trois complaintes paraissent le jour du supplice comme en témoignent les permis d'imprimer chez Valleyre, signés le jour même par le lieutenant général de la police, Lenoir. Ce n'est pas le moindre paradoxe de la complainte : au plus près de l'événement et de l'émotion légitime qu'il suscite, elle développe pourtant un argument définitivement réglé et clôturé. Asservie au pouvoir judiciaire, elle ne donne pas à douter un instant de la culpabilité du supplicié, alors même que dans ce cas précis, Desrues a toujours clamé son innocence. [Cf. illustration 1]

L'air joue un rôle essentiel dans la diffusion des complaintes. Il importe en effet qu'elle s'opère avec la plus grande célérité, ne laissant aucune place à la rumeur, encore moins au risque de soulèvement. ${ }^{(8)}$

A cette fin, la très grande majorité de ces pièces adopte le procédé de composition sur timbre, c'est-à-dire l'usage d'une mélodie préexistante, le plus souvent connue de tous, simple passant ou chanteur confirmé. L'une des trois complaintes de Desrues peut servir d'exemple :

Histoire tragique et morale d'un ci-devant épicier-droguiste Faussaire et empoisonneur Air : Approchez-vous bonorable assistance

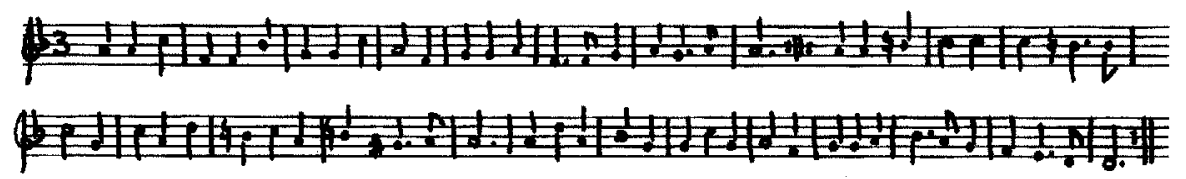

Illustration 2
(8) La diffusion des complaintes de Desrues semble avoir été particulièrement efficace, si l'on en juge par cette remarque de L.-S. MERCIER : "Ainsi, à Paris, tout est matière à chanson; et quiconque, maréchal de France ou pendu, n'a pas été chansonné, a beau faire, il demeure inconnu $d u$ peuple. Je soutiens ici que Desrues, dans les carrefours de la capitale, est plus illustre que Voltaire ", ibid. p. 156. 
(9) Chœur de l'acte II, scène 2 .
Approchez-vous, hommes, femmes et filles

De tous états, et vous aussi garçons ;

Venez frémir des exécrables trames

D’un noir complot forgé par des démons ;

De cette histoire

Qu'on ne peut croire

Le châtiment

Fait preuve assurément.

Le criminel, de qui la Providence

A découvert les tours de son métier,

Reçut d'abord à Chartres sa naissance

Puis dans Paris, un tems, fut Epicier ;

Fit banqueroute,

C'est là la route

De bien des gens

Pour se rendre opulens. [...]

En 1777, il y a déjà plus d'un siècle que cette mélodie est apparue pour la première fois dans l'un des chœurs d'une tragédie lyrique de Lully : Atys (1676). ${ }^{(9)}$ Ce musicien en est très probablement le compositeur. Quelques années plus tard, la mélodie circule déjà sur le PontNeuf, entre les mains de violoneux. En 1723, elle s'associe à la légende de Geneviève de Brabant pour devenir la complainte du même nom. Sous cette forme au moins, cet air s'apprête à connaître une très large diffusion dans tout l'espace francophone. L'imagerie populaire a joué un rôle central dans cette propagation. Lors de l'exécution de Desrues, cet air est sans aucun doute encore connu de beaucoup.

Choisir une mélodie plutôt qu'une autre n'est d'ailleurs pas nécessairement un acte laissé au hasard d'une vogue musicale passagère. Si l'on compare les thématiques de ces deux complaintes, plusieurs motifs semblent communs, par exemple celui de la femme, cruellement et injustement pourchassée, ou encore celui de la solitude devant la mort. 
Après la parenthèse de la période révolutionnaire où peu de complaintes judiciaires sont produites, le XIXème siècle fournit une littérature particulièrement abondante. Sous l'appellation de canard, ${ }^{(10)}$ l'occasionnel connaît alors un succès sans précédent auprès des milieux populaires ${ }^{(1)}$ et se constitue en véritable journal.

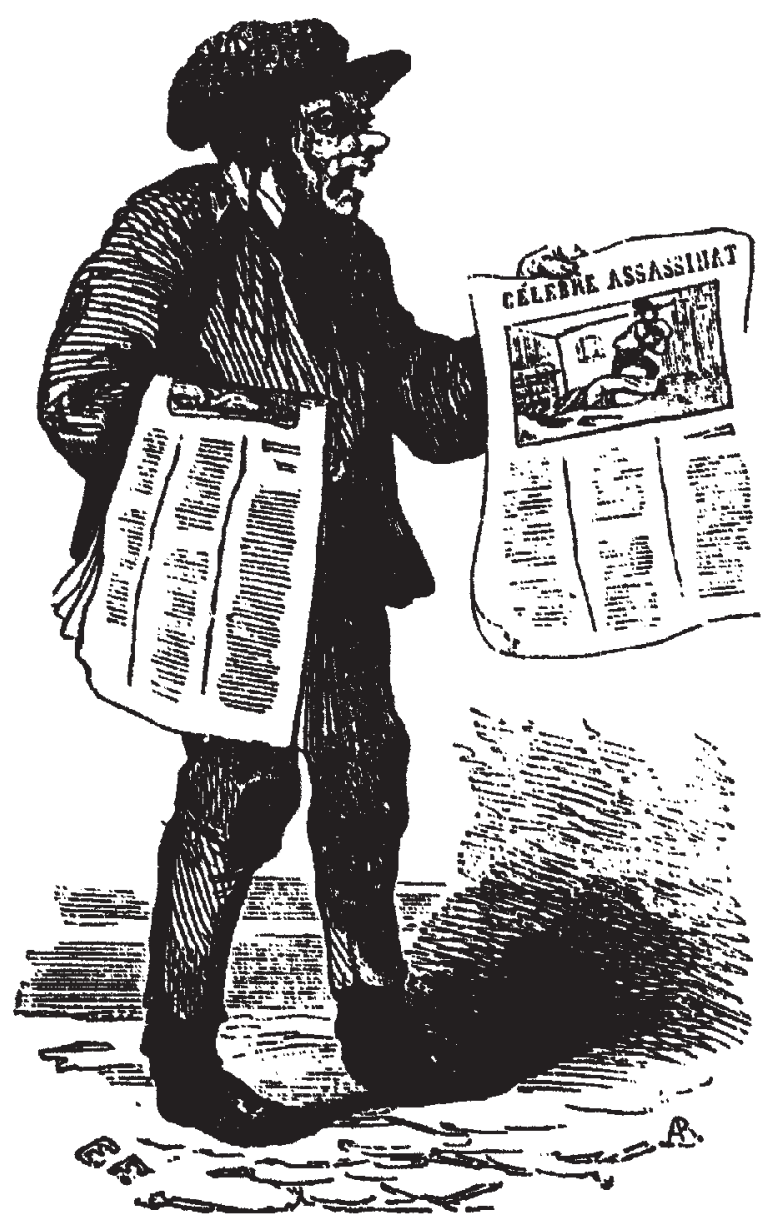

(10) «Vers le début du

XIXème siècle, le mot

"canard", qui fait peut-être allusion à l'aigle impérial figurant sur les bulletins de la Grande Armée, s'applique surtout aux feuilles in-folio, avant de servir à désigner à la fois tous les occasionnels et les nouvelles de faits divers dans la presse périodique ", JeanPierre SEGUIN, Cinq siècles d'imagerie française, Paris, éd. des Musées nationaux, 1973, p. 315.

(11) Principalement citadins.

Illustration 3 
(12) Michel-Louis ROUQUETTE, $L a$ rumeur et le meurtre, l'affaire Fualdès, Paris, PUF, 1992, p. 15.

(13) Frédéric CHAUVAUD, Les criminels $d u$ Poitou au XIXème siècle, La Crèche, Geste éditions, 1999, 359 p.
En 1817, c'est l'assassinat du magistrat Fualdès à Rodez et le très grand retentissement de cette affaire qui serviront de facteurs déclenchant. Deux complaintes ont été composées à cette occasion. L'une deviendra rapidement emblématique : elle se compose de quarante-huit strophes. La tripartition du scénario est encore apparente, mais dans un nouvel équilibre cependant. Une très large place est donnée en effet à la narration des faits, ou du moins à ce que l'on croit en savoir. La rumeur est ici constamment sollicitée, ainsi que toutes sortes d'archétypes et d'imaginaires qui, en définitive, permettent de cerner le type de société auquel elle s'adresse, société qui s'unifie en quelque sorte au travers de la répulsion que provoque cet acte criminel. L'anecdote est alors devenue "exemplaire ", selon l'expression de Michel-Louis Rouquette, "par tout ce qu'elle condense et permet de dévoiler ${ }^{(12)}$ ".

C'est Bastide, le gigantesque, l'Hercule, le géant épouvantable et impassible qui endosse déjà l'habit du monstre froid, ${ }^{(13)}$ avant même d'être un criminel.

C'est Jausion l'insidieux, l'avaricieux, le sanguinaire, prêt à égorger le pauvre Fualdès sur une table de cuisine : monstre lui aussi, mais d'une autre nature.

C'est la femme Bancal la tigresse, qui - inversion particulièrement sordide -, reçoit le sang de Fualdès dans un baquet pour nourrir son cochon.

Ce sont les joueurs de vielle qui, par le jeu assourdissant de leur instrument, étouffent les cris du supplicié et deviennent, à dessein semblet-il, complices de ce tableau criminel. Curieuse mise en abyme, inversée elle aussi, de la fonction du vielleux, l'habituel chanteur des complaintes.

C'est Fualdès l'ami, le confident, le très prudent, le vieillard à l'air aimable, victime innocente suppliant qu'on le laisse mourir en paix avec Dieu : demande refusée par Bastide.

Ce sont des lieux également : l'infâme repaire de la maison Bancal, lieu de bacchanale et de prostitution, l'asile du crime.

C'est enfin l'impénitence de deux condamnés (sur trois), et donc la vision d'horreur d'un châtiment éternel, sans rémission possible. 
Même s'ils ont pu confier peut-être à Desaugiers, Berryer et Catalan le soin de composer cette complainte, "l'aimable président » du tribunal, représentant de la clémence royale ou le "digne confesseur " constituent autant de commanditaires possibles de cette tragédie mise en musique. L'horreur qu'elle inspire rassemble dans une même indignation femmes et hommes de tous milieux. Dans de telles circonstances en effet, ainsi que le remarque Frédéric Chauvaud : "Le pouvoir trouve là une occasion privilégiée de renforcer l'idéologie qui le sert et dont il s'inspire : nier les disparités sociales et les oppositions politiques, réaffirmer l'universalité de la nature bumaine, le primat de la morale et l'éternel combat du bien et du mal. ${ }^{(14)}$ "

\section{[Cf. illustrations 4 et 5. - La complainte de Fualdès]}

Cette complainte semble à bien des égards inaugurer une ère nouvelle qui, parallèlement au succès du canard et au développement du journalisme, délaisse la confession au profit du compte-rendu d'audience et de la rumeur. Il s'en faut de beaucoup qu'elle ait vocation à être lue par le plus grand nombre. Pour quatre-vingt pour cent de la population française, elle ne peut qu'être écoutée, souvent regardée et parfois mémorisée pour partie. Le public est particulièrement attentif si l'on en juge par cette description d'un chanteur de complaintes en 1869 :

"Monté sur un escabeau, il faisait à la foule tremblante le récit d'un drame terrible. Une grande toile, partagée en quatre ou six petits carrés, représentait les différentes phases du crime. On y voyait, largement brossés, les assassins munis de longs couteaux et les malheureuses victimes aux abois [...]. ${ }^{(15)}$ "

Dans cette stratégie de diffusion, et dans le sillage d'une tradition solidement établie, l'air joue une fois encore un rôle essentiel. La mélodietimbre apparaît vers 1760, associée à une complainte sur la mort du maréchal de Saxe. On a pu l'entendre également lors de la disparition de Hoche (1797) ou de celle du duc de Montebello (1809). D'après le catalogue de timbres de Patrice Coirault, ${ }^{(16)}$ son usage semble néanmoins assez peu fréquent. L'appliquer à l'affaire de Rodez n'est donc peut-être pas innocent. Par le choix d'une mélodie en quelque sorte connotée, Fualdès devient implicitement le grand homme dont on pleure la disparition.
(14) Ibidem, p. 73.

(15) Ernest SCHNAI-

TER, Le Clocher, 9 octobre 1869.

(16) Bibliothèque nationale de France, département de la Musique. 


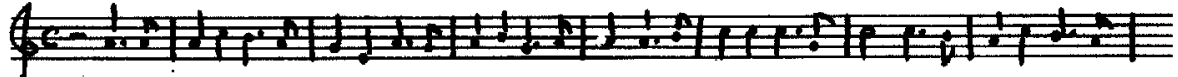 12
}

\author{
Illustration 4. - La complainte de Fualdes.
}

\section{VÉRITABLE COMPLAINTE}

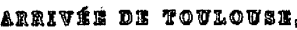

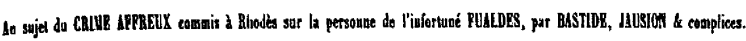

\begin{tabular}{|c|c|c|c|}
\hline 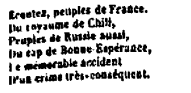 & 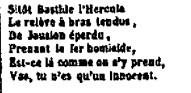 & at anine & 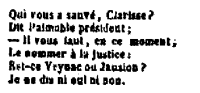 \\
\hline 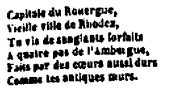 & 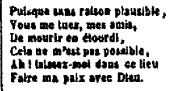 & sist. & 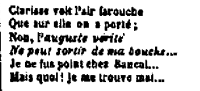 \\
\hline 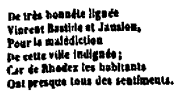 & 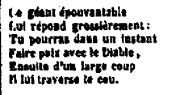 & 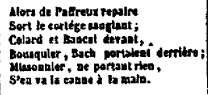 & 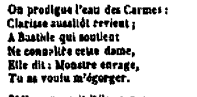 \\
\hline 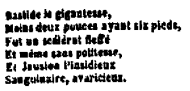 & 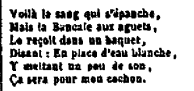 & 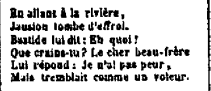 & 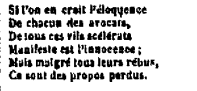 \\
\hline 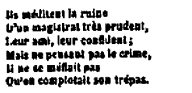 & 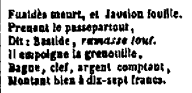 & 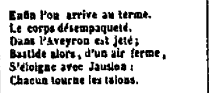 & tart \\
\hline 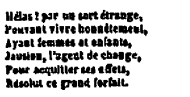 & 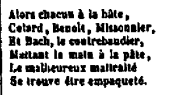 & 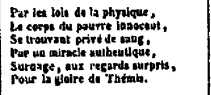 & istosits \\
\hline 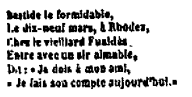 & 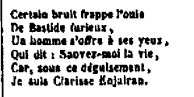 & 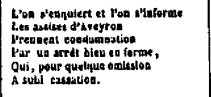 & 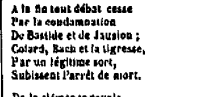 \\
\hline 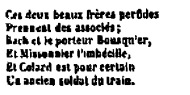 & 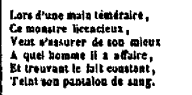 & 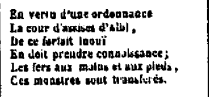 & $\begin{array}{l}\text { athoum } \\
\text { ind } \\
\text { ond } \\
\text { ind }\end{array}$ \\
\hline 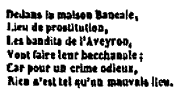 & 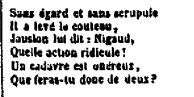 & inters. & int, \\
\hline $\begin{array}{l}\text { the } \\
\text { Tal; } \\
\text { at } \\
\text { uche; }\end{array}$ & 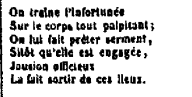 & 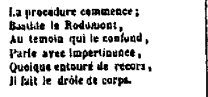 & stis \\
\hline 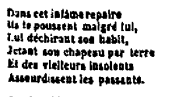 & 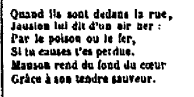 & 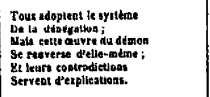 & 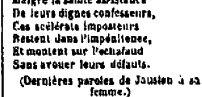 \\
\hline 1. & 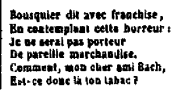 & 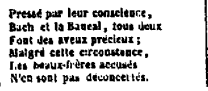 & 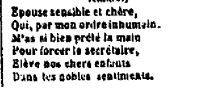 \\
\hline
\end{tabular}

Illustration 5. - La complainte de Fualdes. 
La répercussion de cette complainte fut très importante et, aujourd'hui encore, nos musiques traditionnelles peuvent aisément en témoigner. Bien d'autres complaintes judiciaires, mais également des chansons (pour certaines très légères), des cantiques mêmes, ont été composés ou ont trouvé une nouvelle jeunesse au contact de ce timbre. L'air est tellement connu qu'il rassemble aisément et efficacement, tant à la veillée, qu'à la procession ou au repas de noces.

Très tôt, le nom de Fualdès remplacera celui du maréchal de Saxe dans la désignation du timbre. La référence à l'affaire disparaîtra ellemême, conférant ainsi à la mélodie une totale autonomie. La mention "sur l'air de toutes les complaintes (17)", suffira désormais à la reconnaittre.

Quelques autres mélodies dont la célèbre Paimpolaise, serviront ultérieurement de support aux nouvelles créations, s'inscrivant ainsi dans l'exigence d'actualité inhérente à ce genre. Mais le temps est au déclin en ce XXème siècle commençant. Certes les marchands de chansons ne quitteront pas de sitôt l'espace des foires locales, mais à la complainte criminelle, on préférera plus souvent les dernières chansons en vogue à Paris. En 1925 encore, à la foire de Gaitres, on pouvait acquérir la «complainte de la tuerie du Grand Moulin (Ougnolles) (18) ", bientôt on chantera également l'affaire Violette Nozières, le crime d'Oradour-sur-Glane. La concurrence de la presse, l'apparition de nouveaux médias feront taire les diseurs de chansons.
(17) Voir par exemple la complainte historique sur la sorcière du vieux Bel-air lors de son séjour à Nantes : sur l'air de toutes les complaintes..., collection GUERAUD, Bibl. municipale de Nantes, ms. 2224, p. 103.

(18) Pièce publiée dans La Liborne, Revue du Cerdo, t.1, 1994, $\mathrm{n}^{\circ} 2$, p. 97. Je remercie Jean-Louis NEVEU, documentaliste du Cerdo de me l'avoir signalée. 